disease duration 13.1 years). Pulmonary lesions were found in $146(70.2 \%)$ of RA patients before treatment Imaging findings were 81 of ILD (39\%), 45 of nodular lesion (21.6\%) and 115 of AD (55\%). Cluster analysis showed 6 clustered (Fig.), 1; no pulmonary lesions, 2; AD without bronchoectasia, 3; AD with bronchoectasia, 4; AD with curved linear opacities, 5; AD with nodular lesions, and 6; reticular pattern with AD.

AD was common abnormalities and coexisted with other pulmonary lesions in RA. AD was found in $79 \%, 78 \%$ and $71 \%$ of patients with pulmonary abnormalities, ILD and nodular lesions, respectively. AD alone, AD with ILD, and AD with nodular pattern were found in $16.3 \%, 8.6 \%$ and $28.9 \%$, respectively, while patients without pulmonary lesions were $29.8 \%$ in RA. AD was frequently associated with ILD and nodule compare to non-AD.

No differences were found in gender, smoking history, disease duration and disease activity between patients with and without AD. New emergence or exacerbation of pulmonary abnormalities developed in $A D$ patients compared to those without pulmonary abnormalities or AD. No significant differences were found in clinical features, among $A D$ alone, $A D$ with ILD and $A D$ with nodules.

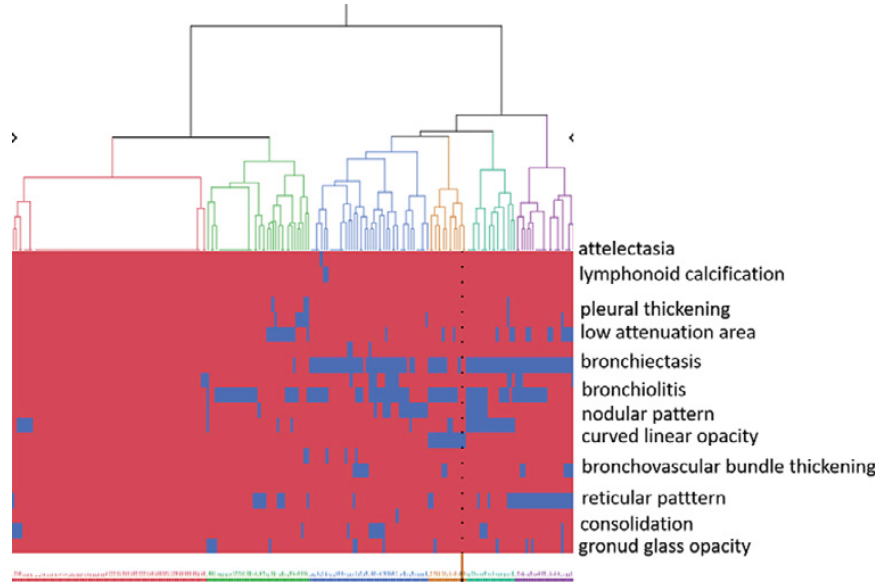

Conclusions: Pulmonary abnormalities were found in $70 \%$ in RA. AD was found in $55 \%$ of RA patients and coexisted with other pulmonary lesions such as ILD and nodular lesions. Patients with AD frequently showed newly emerging or worsening pulmonary lesions, regardless of the coexistence of other pulmonary lesions. Thus, AD is shared and critical pulmonary abnormality in RA.

Disclosure of Interest: None declared

DOI: 10.1136/annrheumdis-2017-eular.5828

\section{FRI0135 TREATMENT OF JAPANESE EARLY RHEUMATOID ARTHRITIS PATIENTS WITH LOW-DOSE PREDNISOLONE FOR MAXIMUM 1 YEAR LEADS TO EARLIER IMPROVEMENT OF DISEASE ACTIVITY AND DOES NOT WORSEN BONE METABOLISM STATUS AND RATES OF NEW COMPLICATIONS}

\section{E. Torikai, M. Suzuki, Y. Matsuyama. Orthopaedic Surgery, Hamamatsu} University School of Medicine, Hamamatsu, Japan

Background: Glucocorticoid (GC) therapy for rheumatoid arthritis patients improves joint inflammation and destruction; however, it is associated with risk of complications such as osteoporosis, diabetes (DM), and cardiovascular (CV) disease. Although EULAR recommends that low-dose GC should be administered for up to 6 months, the ideal dose and duration of GC use remain unresolved.

Objectives: To investigate the efficacy and safety of low-dose GC therapy in addition to other disease-modifying antirheumatic drugs (DMARDs) for maximum 1 year in Japanese early RA patients.

Methods: Ninety-six Japanese RA patients with disease duration of $<2$ years were included. Patients were treated with a T2T strategy; if disease activity did not improve within 3 months, their DMARDs were replaced with alternatives or additional DMARDs were added. We excluded patients with a history of prior complications, including CV disease, DM, or vertebral fracture. We classified patients into two groups, one was group treated with DMARDs alone ( $N$ group; 35 females and 10 males) and the other with maximum $5 \mathrm{mg}$ of $\mathrm{GC}$ for maximum 1 year along with DMARDs (GC group; 40 females and 11 males). The mean ages of the $\mathrm{N}$ and GC groups were 56.3 and 60.9 years, respectively. Thirty-four percent of patients were treated with MTX monotherapy, $20.9 \%$ were treated with combined conventional synthetic DMARD with MTX, and $31.3 \%$ were treated with a biological agent. Regarding MTX or biological agent use rates, no significant statistical differences were observed between the groups. We evaluated the change of DAS28-CRP scores for 3 years, bone metabolism makers [urine type I collagen cross-linked $\mathrm{N}$-telopeptide (NTX), serum tartrate-resistant acid phosphatase 5b (TRACP5b), serum bone-specific alkaline phosphatase (BAP), and serum osteocalcin (OC)], bone mineral density (BMD) of lumber spine (L-spine) and femoral neck (FN) and the rate of new complications. Comparisons of BMD and the rate of new complications were made at baseline and 3 years after initiating GC treatment. Results: There were no significant differences in DAS28-CRP scores at baseline.
In the GC group, the mean GC dose was $2.46 \mathrm{mg} /$ day. At 1 month after treatment, there was a significant difference in the improvement rate of DAS28-CRP scores in the GC group compared with the $\mathrm{N}$ group. However, no significant difference was observed between the two groups at 3 months or more post-treatment (Fig.1). None of the bone metabolism makers and BMD deteriorated in the GC group and there were no statistical differences between both groups (Table.1, Fig.2). New complications occurred in four cases in the $\mathrm{N}$ group (one, vertebra fracture; one, CVD; and two, high HbA1c levels) and four cases in the GC group (two, vertebrae fractures and two, high $\mathrm{HbA1c}$ levels). There were no significant differences in the rate of new complications between both groups.
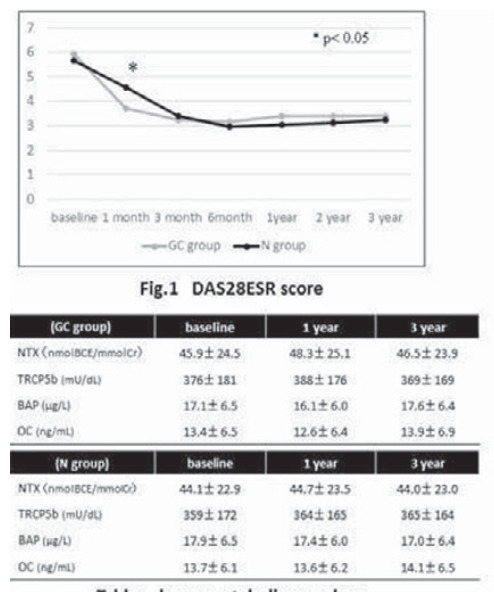

Table. bone metabolism makers
Fig.1 DAS28ESR score

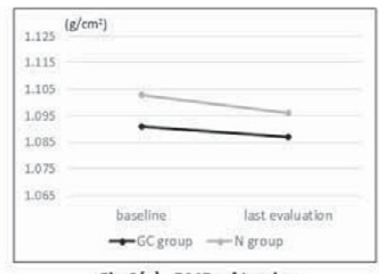

Fig.2(a) BMD of L-spine

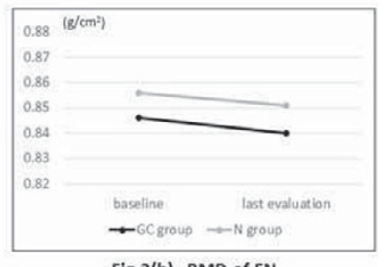

Fig.2(b) BMD of FN
Conclusions: The treatment of early rheumatoid arthritis by low-dose GC for maximum one year enables earlier improvement of disease activity and does not worsen bone metabolism status or the rate of new complications. The therapy does not pose a problem in the middle term. This study confirms that use of GC in RA patients leads to patient satisfaction.

Disclosure of Interest: None declared

DOI: 10.1136/annrheumdis-2017-eular.2092

\section{FRI0136 OVARIAN RESERVE, AS ASSESSED BY MEASURING SERUM ANTI-MÜLLERIAN HORMONE LEVELS, DECLINES MORE RAPIDLY OVER TIME IN RHEUMATOID ARTHRITIS PATIENTS COMPARED TO CONTROLS}

J. Brouwer $^{1,2}$, J.S. Laven ${ }^{1}$, J.M. Hazes ${ }^{2}$, N.S. Erler ${ }^{3}$, J.A. Visser ${ }^{4}$ R.J. Dolhain ${ }^{2} .{ }^{1}$ Division of Reproductive Medicine - Department of Obstetrics and Gynaecology; ${ }^{2}$ Department of Rheumatology; ${ }^{3}$ Department of Biostatistics; ${ }^{4}$ Department of Internal Medicine, Erasmus MC University Medical Center Rotterdam, Rotterdam, Netherlands

Background: The ovarian reserve in women with rheumatoid arthritis (RA) may be compromised, based on a reduced fertility ${ }^{1}$ and a younger age at menopause ${ }^{2}$. Serum anti-Müllerian hormone (AMH) levels are a proxy for the ovarian follicle pool, and are the most reliable predictor of the age at which menopause sets in. Objectives: Our objectives were to study the intra-individual change in $\mathrm{AMH}$ levels in female RA patients, and to study the effect of RA-related factors on the decline of $\mathrm{AMH}$ levels over time.

Methods: Female RA patients from a nationwide prospective cohort study (PARA study) in 2002-2008, were re-assessed in 2015-2016. Serum AMH levels were measured using the pico AMH assay (provided by Ansh Labs, Texas, USA) and compared to healthy controls (Lie Fong, 2012) ${ }^{3}$. A linear mixed model was built to assess the effect of RA-related clinical factors on the decline of serum AMH levels over time.

Results: 128 women were re-assessed at a mean age of $42.6 \pm 4.4$ years, with a median disease duration of 15.8 (IQR 12.7-21.5) years. The participants appeared a more fertile selection of the original PARA cohort. The mean time between the first and the follow-up assessments was $10.7 \pm 1.8$ years. At follow-up, more patients had $\mathrm{AMH}$ levels below the 10th percentile of controls $(39 \%$; $95 \%$ $\mathrm{Cl} 31-48 \%)$, than at baseline $(16 \% ; 95 \% \mathrm{Cl} 9.3-22 \%)$. The linear mixed model showed only a significant effect of age, and no significant effect of RA-related factors on the decline of serum AMH levels over time.

Conclusions: This is the first longitudinal study on $\mathrm{AMH}$ levels in women with $\mathrm{RA}$, and is showing that $\mathrm{AMH}$ levels in RA patients decline more rapidly over time compared to healthy controls. This indicates that the disease process of RA has a negative impact on the ovarian reserve of young pre-menopausal RA women.

\section{References:}

[1] Brouwer J, Hazes JM, Laven JS, Dolhain RJ. Fertility in women with rheumatoid arthritis: influence of disease activity and medication. Ann Rheum Dis 2015;74:1836-41.

[2] Del Junco D. The relationship between rheumatoid arthritis and reproductive function [Dissertation]. Houston, Texas: The University of Texas Health Sciences Center at Houston School of Public Health; 1988. 